\title{
Overexpression of S-adenosylmethionine decarboxylase (SAMDC) in Xeno-pus embryos activates maternal program of apoptosis as a "fail- safe" mechanism of early embryogenesis
}

\author{
Masatake Kal ${ }^{1, *}$, Chikara Kalto ${ }^{1, * *}$, Hiroshi Fukamach ${ }^{1}$, Takayasu Higo ${ }^{1, * * *}$, ElJI Ta-Kayama ${ }^{2}$, Hiroshi \\ Hara $^{3}$, Yoshikazu Ohya ${ }^{4}$, KazUeI Igarashi ${ }^{5}$, Kolchiro ShIOKaWa ${ }^{1, * * *}$ \\ 1 Laboratory of Molecular Embryology, Department of Biological Sciences, Graduate School of Science, \\ University of Tokyo, Hongo 7-3-1, Bunkyo-ku, Tokyo, 113-0033, Japan \\ 2 Department of Parasitology, National Defense Medical College, Namiki 3-2, Tokorozawa, Saitama, 359-8543, \\ Japan \\ 3 Medicinal Research Laboratories, Taisho Pharmaceutical Co., Ltd., 1-403, Yoshino-cho, Kita-ku, Saitama \\ 331-9530, Japan \\ 4 Department of Integrated Biosciences, Graduate School of Frontier Sciences, University of Tokyo, 5-1-5 \\ Kashiwanoha, Kashiwa, Chiba 277-8562, Japan \\ 5 Faculty of Pharmaceutical Sciences, Chiba University, Yayoi-eho 1-33, Inage-ku, Chiba 263-8522, Japan
}

\begin{abstract}
In Xenopus, injection of S-adenosylmethionine decarboxylase (SAMDC) mRNA into fertilized eggs or 2-cell stage embryos induces massive cell dissociation and embryo-lysis at the early gastrula stage due to activation of the maternal program of apoptosis. We injected SAMDC mRNA into only one of the animal side blastomeres of embryos at different stages of cleavage, and examined the timing of the onset of the

apoptotic reaction. In the injection at 4- and 8-cell stages, a considerable number of embryos developed into tadpoles and in the injection at 16- and 32-cell stages, all the embryos became tadpoles, although tadpoles obtained were sometimes abnormal. However, using GFP as a lineage tracer, we found that descendant cells of the blastomere injected with SAMDC mRNA at 8- to 32-cell stages are confined within the blastocoel at the early gastrula stage and undergo apoptotic cell death within the blastocoel, in spite of the continued development of the injected embryos. These results indicate that cells overexpressed with SAMDC undergo apoptotic cell death consistently at the early gastrula stage, irrespective of the timing of the mRNA injection. We assume that apoptosis is executed in Xenopus early gastrulae as a "fail-safe" mechanism to eliminate physiologically-severely damaged cells to save the rest of the embryo.
\end{abstract}

Key words: SAMDC overexpression, maternal apoptosis program, processing-defective SAMDC mutant, $M B T$, fail-safe mechanism.

\footnotetext{
${ }^{*}$ Corresponding author, Dr. Masatake KAI, Department of Anatomy and Developmental Biology, University College London, Gower Street, London, WC1E 6BT, United Kingdom. E-mail: m.kai@ucl. ac.uk

**Laboratory of Developmental Biochemistry, Graduate School of Pharmaceutical Sciences, University of Tokyo, Hongo 7-3-1, Bunkyo-ku, Tokyo 113-0033, Japan
}

***Department of Basic Medical Sciences, The Institute of Medical Science, University of Tokyo, 4-6-1 Shirokanedai, Minato-ku, Tokyo 108-8639, Japan

****To whom reprint requests should be addressed. Department of Biosciences, School of Science and Engineering, Teikyo University, Toyosatodai 1-1, Utsunomiya, Tochigi 320-8551, Japan Received April-4-2003 Revised May-I-2003 Accepted May-13-2003 


\section{INTRODUCTION}

In Xenopus embryogenesis, cleavage proceeds rapidly without $G_{1}$ phase in the cell cycle, and during the cleavage stage cells do not synthesize much RNA[1], since informational molecules necessary for this early phase of development are provided mainly as maternal mRNAs and preformed proteins $[2,3]$. When embryos reach the late blastula stage, $G_{1}$ phase appears in the cell cycle[4], and important changes in cellular activities, which are collectively called MBT (midblastula transition), take place before embryos start gastrulation[5]. The main changes which take place here are transcriptional activation[1, 5, 6], shift from the synchronous to asynchronous cell division, and commencement of cell movement[5, 7, 8]. Along with these changes, transcription of ribosomal RNA genes is also initiated[9], and definitive nucleoli start to appear as the cytological manifestation of the rRNA synthesis[10].

Recently, we performed experiment to inject S-adenosylmethionine decarboxylase (SAMDC) mRNA into Xenopus fertilized eggs, and found that the overexpression of SAMDC in Xenopus cleavage stage embryos induces cell dissociation shortly after MBT[11], due to activation of the maternal program of apoptosis[12]: The dissociated cells were TUNEL positive, contained fragmented nuclei and the fragmented DNA, and the effect of SAMDC mRNA injection was abolished by $\mathrm{Bcl}-2$ mRNA injection[12]. Since the whole effect of SAMDC mRNA injection was canceled by coinjection of SAM (S-adenosylmethionine), the apoptosis induced was concluded to be due to the deficiency of SAM.

While we were performing these experiments, similar apoptotic reaction was found to take place in Xenopus embryos when they were treated with ã-ray [13, 14], hydroxyurea[15], cycloheximide[14, 15] and á-amanitin [14, 16] during cleavage stage. The apoptotic cell death was also found to occur in normally-developing Xenopus embryos[17], although the site of the appearance of apoptotic cells or the number of the apoptotic cells differed from embryo to embryo. More recent studies report that the apoptotic reaction can be observed in embryos altered in DNA methylation[18, 19], defective in endogenous function of XChkl kinase[20] or blocked DNA replication[20]. A similar apoptotic reaction has been found to take place also in zebrafish embryos when they were treated with hydroxyurea, aphidicolin or camptothecin during the cleavage stage [21].

An interesting aspect of the programmed apoptotic reaction found in Xenopus embryos is that it takes place constantly during the late blastula to early gastrula stages and never before [11-16, 18-20, 22]. In the previous study, we have tested a wide dosage range of SAMDC MRNA ( 0.1 pg to $10 \mathrm{pg}$ ) and found that when the "apoptosis-induced" developmental arrest took place it always took place at the early gastrula stage[11]. Furthermore, it has been suggested that DNA damage response is an element of MBT, and Xenopus embryos have multiple mechanisms to block the apoptotic pathway after the MBT[22]. These results strongly suggest that the apoptotic program may be regulated by a clock mechanism.

In this study, we intended to know how and when the apoptosis takes place when SAMDC mRNA was injected at different stages of cleavage. To address this issue, we injected here SAMDC mRNA into only one blastomere at cleavage stages later than 2-cell stage. By doing this experiment, we also expected to know how embryos behave when the apoptosis was induced in a relatively small population of cells within the embryo. We found here that almost all the embryos injected with SAMDC mRNA at later stages (8- to 32-cell stages) develop into tadpoles without showing any sign of cell dissociation at the early gastrula stage at least in their outer appearance. This was at first not easy to understand, since we expected that the apoptosis should take place shortly after the MBT irrespective of the stage of the mRNA injection. In such apparently normally developing embryos, however, we found that cells which received SAMDC mRNA overexpress SAMDC and are dissociated at the early gastrula stage to disappear from the embryo after being confined within the blastocoel. Based on these results, we suggest here that in Xenopus embryos the maternal program of apoptosis constitutes a failsafe mechanism of early embryogenesis, which eliminates physiologicallyseverely damaged cells to save the rest of the embryo. 


\section{MATERIALS AND METHODS}

\section{Plasmids and in vitro transcription of $m R N A s$}

pSP36T-XSD3, which contained the wild-type Xenopus SAMDC cDNA (XSD3)[23], was maintained and used as described[11, 12].

A processing-defective Xenopus SAMDC mutant CDNA was newly constructed from XSD3 by replacing the serine residue with alanine at the putative reaction center (at residue 70)[23, 24]. Primer pairs used in $P C R$ to prepare the 5 '-half of the mutant ORF were SDF1 (5'-CGA TCG ATA TCA TGA AGA TGG AGG AGA GC-3') and SDR2 ( $5{ }^{-}{ }^{-}$-CAA ACG AGA CCA GAT CTG GTC TCT GAG TAC ATA ACG TTC C-3') underlined are Bsal sites), and those used to prepare the 3'-half of the mutant ORF were SDF2 (5'-CTC AGA GAC CAG ATC TGG TCT CGT TTG TCT CCA AGA GAC G-3'; underlined are Bsal sites) and SDR1 (5'-TCC CCG CGG TTG CTA GCT CTG CTG TGG CTG GAT C-3')[11]. To ligate the 5'-half and the 3'-half of the mutated ORF, the above PCR products were subjected to second $P C R$ for five cycles, then to further cycles using SDF1 and SDR1 as new primer pairs. The PCR product finally obtained was inserted into pTD315 at BspHI and Nhel sites (pTD315-XSD3-R). A double-stranded DNA fragment (with 5'-protruded ends) was prepared by annealing two single-stranded oligo nucleotides, 5'-ACT CAG TGA GGC TAG CAT G-3' and 5'-CAA ACA TGC TAG CCT CAC T-3' (boldfaces are the mutated bases), and this was inserted into pTD315-XDS3-R at Bsal sites. The sequence of the DNA obtained was confirmed, subcloned into pSP36T at $\mathrm{Ncol}$ and Xbal sites \{pSP36T-XSD3(S70A)\} and used as the template for the mutated SAMDC.

Plasmids carrying the wild-type human SAMDC (pCM9) and the processing-defective human SAMDC (S68A) cDNAs (in which serine at the reaction center was replaced by alanine) were used as described by[25]. GFP mRNA was obtained from pbGFP/RN3P as described[26].

All the DNA constructs were linearized at the Pvull or Notl site, and transcribed in vitro with SP6 RNA polymerase (Ambion, Austin, TX, USA) in the presence of a cap analog, $m^{7} G\left(5^{\prime}\right) p p p$ $\left(5^{\prime}\right) \mathrm{G}$ (New England Biolabs, Beverly, MA, USA). Sizes of all the in vitro transcribed RNAs were confirmed by gel electrophoresis.

\section{Microinjection of $m R N A$}

Unfertilized eggs of Xenopus laevis were manually ovulated from gravid females which had been injected with a human chorionic gonadotropic hormone, Gonatropin (Teikoku Zoki Co., Tokyo, Japan). Eggs were artificially fertilized, and dejellied in $2 \%$ cysteine-HC1 ( $\mathrm{pH} 8.0)[27]$.

mRNAs dissolved in distilled water were microinjected into embryos as specified in each experiment in I $\times$ modified Barth's solution (MBS), containing $3 \%$ Ficoll 400 and $50 \mu \mathrm{g} / \mathrm{ml}$ gentamycin. Injected embryos were kept in I $\times$ MBS until stage 7[28], then transferred into either $0.1 \times$ or $1 \times$ Steinberg's solution as indicated in each experiment. Embryos were cultured at $21-23^{\circ} \mathrm{C}$ throughout experiments.

\section{Light and electron microscopic observations}

For light microscopic observations, embryos were fixed for $2 \mathrm{~h}$ with MEMFA (0.1 M [pH 7.4], 2 mM EGTA, 1 mM MgSO $4.3 \%$ formaldehyde), dehydrated in a graded series of ethanol, and embedded in paraffin. Samples were sectioned serially at $5 \mu \mathrm{m}$,stained with hematoxylin and eosin, and examined under a light microscope [11].

For transmission electron microscopy, embryos were fixed overnight in $2.5 \%$ glutaraldehyde in $0.1 \mathrm{M}$ Cacodylate buffer $(\mathrm{pH} 7.4)$ at $4^{\circ} \mathrm{C}$. They were post-fixed with $1 \% \mathrm{OsO}_{4}$ for $2 \mathrm{~h}$, dehydrated in a graded series of ethanol, embedded in Embedding Resin (TAAB, Aldermaston, UK), and sectioned at $90 \mathrm{~nm}$. Sections were stained with uranyl acetate and lead citrate, and examined in an electron microscope (100CX; JEOL, Tokyo, Japan)[12]

\section{Assays for SAMDC activity}

Embryos were homogenized in $50 \mathrm{mM}$ HEPES-KOH (pH 7.4), containing $50 \mathrm{mM} \mathrm{KCl}, 0.1 \mathrm{mM}$ EDTA, $2.5 \mathrm{mM} \mathrm{DTT}, 10 \%$ glycerol and $20 \mathrm{mM}$ Futhan (Torii Pharmaceutical, Tokyo. Japan). Soluble fractions, obtained by centrifugation at $16,000 \mathrm{rpm}$ for $10 \mathrm{~min}$, were dialyzed twice for $2 \mathrm{~h}$ at $4^{\circ} \mathrm{C}$ against the homogenizing buffer. Samples were incubated with $2 \mathrm{mM}$ putrescine and $0.2 \mathrm{mM}$ of (carboxyl-14C)SAM (2.6 kBq/mmole) (Perkin Elmer, Wellesley, MA, USA) at $37^{\circ} \mathrm{C}$ for $1 \mathrm{~h}$. Reaction was stopped by adding an equal volume of $2 \mathrm{M}$ citric acid at $37^{\circ} \mathrm{C}$, and release of ${ }^{14} \mathrm{CO}_{2}$ was measured as described previously[23].

\section{RESULTS}

We previously showed that SAMDC mRNA injection into 1- or 2-cell stage Xenopus embryos induces deleterious cell dissociation followed by developmental arrest due to apoptosis shortly after MBT[12]. We injected here 20, 10 and $5.0 \mathrm{nl}$ of SAMDC mRNA solution (all at $0.1 \mathrm{ng} / \mathrm{nl}$ ) into the animal top portion of one of the blastomeres of 1-, 2- and 4-cell stage embryos, respectively. Using the same batch of embryos, we also injected 2.5, 1.3 and $0.63 \mathrm{nl}$ of the same SAMDC mRNA solution into one of the animal top blastomeres of 8-, 16- and 32-cell stage embryos, respectively. These experimental designs are shown diagrammatically in Fig 1 (left half). Since the volume of an egg is roughly $1 \mu \mathrm{l}$, the amount of SAMDC mRNA injected here was approximately $2 \mathrm{ng}$ per $\mathrm{u} 1$ of the cytoplasm within the injected blastomere at 1-, 2- and 4-cell stages. We assume that the concentration of SAMDC mRNA in the blastomeres of embryos injected at stages later than 4-cell stage was slightly larger than $2 \mathrm{ng} / \mathrm{nl}$, since animal top blastomeres are usually smaller than vegetal side blastomeres in these stages.

When we cultured these SAMDC mRNA-injected embryos in $0.1 \times$ Steinberg's solution, all the embryos injected at 1 - and 2-cell stages underwent cell dissociation at the early gastrula stage (stage $10.5 ; 12.5 \mathrm{~h}$ postfertilization) and autolyzed (Fig 1) due to osmotic 


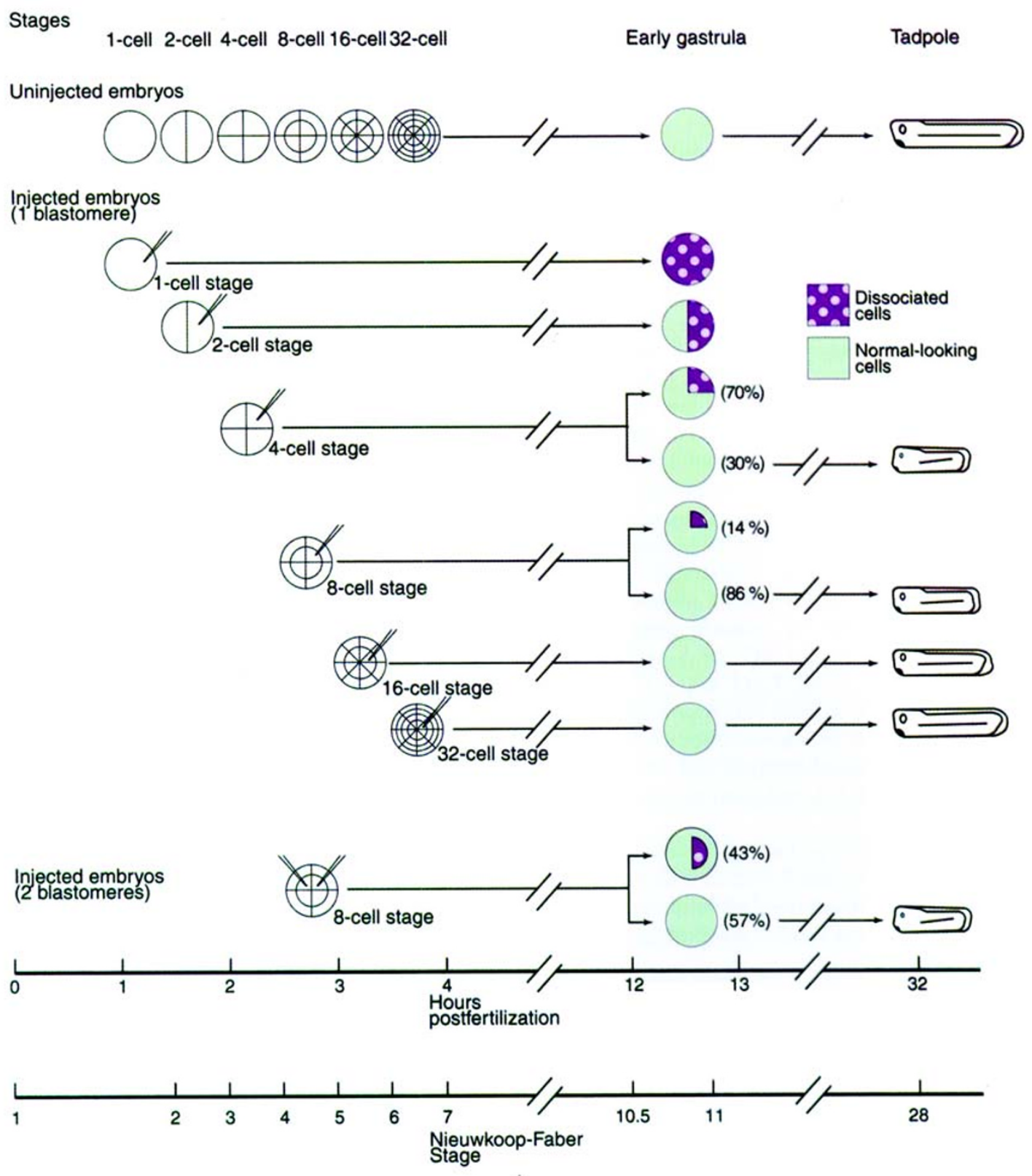

Fig1. Experimental designsand results of theexperimentsto injectSAMDCmRNAatdifferentstages of cleavage. We injected differentvolumes of SAMDC mRNA solution $(20,10,5.0,2.5,1.3$ and $0.63 \mathrm{nl}$; all at $1 \mathrm{ng} / \mathrm{nl})$ into only one of the animal top blastomeres at different stages (respectively, 1-, 2-, 4-, 8-, 16-and 32-cell stage, or 1.25, 1.75, 2.25, 2.75, 3.15and3.75h post-fertilization) or into two animal top blastomeres (2. $5 \mathrm{nl}$ of $1 \mathrm{ng} / \mathrm{nl}$ solution to each blastomere) at 8 -cell stage ( $2.75 \mathrm{~h}$ post-fertilization) (bottom). In these experiments, we did not distinguish the presumptive dorsal orventral blastomeres. All embryos were keptcultured in $0.1 \times$ Steinberg's solution afterstage6. Allembryos injected at 1 -or 2-cell stages underwent cell dissociation and autolyzed due to osmotic shock at the early gastrula stage (stage 10.5). Embryos injected with SAMDC mRNA into one of the blastomeres at 4- and 8-cell stages either arrested development at the early gastrula stage (70\% and $14 \%$, respectively)ordeveloped into tadpoles ( $30 \%$ and $86 \%$, respectively). All theembryos injectedat 16 -and 32 -cell stages developed intotadpoles. In embryos injected with SAMDC mRNA into two animal side blastomeres at 8-cell stage, $43 \%$ of the embryos underwent cell dissociation and arrested development, butthe rest $57 \%$ developed intotadpoles. Some of the tadpoles developed from SAMDC mRNA-injected embryos were normal, butsome were abnormal. To the right of the figure, only abnormal tadpoles are drawn schematically, reflecting the extent of theanomaly in their outer appearance. In all cases, one experimental group consisted of at least 20 embryos. 
shock (see [11]). The dissociated cells which appeared here were TUNEL positive, contained the "ladder'-forming DNA, and the cell dissociation was suppressed by Bcl-2 (data not shown, but see [12]), confirming that the cell dissociation is due to the execution of the maternal program of apoptosis. In embryos injected with SAMDC mRNA at 4- or 8-cell stage, however, cell dissociation accompanied with embryo death took place only in $70 \%$ or $14 \%$ of the embryos, respectively. The rest of the embryos developed into tadpoles without showing any sign of cell dissociation at least in their outer appearance (Fig 1), although they showed some abnormality in their morphology. In embryos injected at 16- or 32-cell stage, cell dissociation was totally not observed at least in their outer appearance, and all the injected embryos developed into tadpoles, although here again they were more or less abnormal. Roughly speaking, the earlier the stage of the injection, the larger the extent of the anomaly, and the anomaly included small head (sometimes acephaly), small trunk and tail, bending of body axis, and shortening of the body length (see also Fig 4).

We injected $2.5 \mathrm{nl}$ each of the above-used SAMDC mRNA solution $(0.1 \mathrm{ng} / \mathrm{nl})$ into two animal-side blastomeres at the 8-cell stage (the concentration was ca. $0.25 \mathrm{ng} /$ blastomere or slightly larger) (Fig 1, bottom). In this case, as much as $43 \%$ of the injected embryos underwent cell dissociation and died at the early gastrula stage: The rest $57 \%$ of the embryos developed into tadpoles, Mthough again their morphology was sometimes abnormal. Thus, embryos injected with SAMDC mRNA into two animal blastomeres at the 8- cell stage developed like the embryos injected with the mRNA into only one blastomere at the 4-cell stage, suggesting that it is the total volume of SAMDC mRNAinjected cytoplasm per embryo that determines whether embryos undergo massive cell dissociation or continue development.

SAMDC activity increases in SAMDC mRNA-injected embryos irrespective of the timing of the mRNA injection

We repeated the experiment in Fig 1 using embryos at 2-, 8- and 32-cell stages. In this experiment, all the embryos injected with SAMDC mRNA at the 2-cell stage stopped development at the early gastrula stage, whereas all the embryos injected with SAMDC mRNA at the 32-cell stage and $80 \%$ of embryos injected at the 8 -cell stage developed into tadpoles. At the blastula stage, however, we detected substantial increases in SAMDC activity in all the three groups of these embryos (Tab 1). We then normalized the SAMDC activities according to the estimated volume of the injected blastomere, and obtained SAMDC activities which were large enough to induce cell dissociation for all the three injection groups (144 to 320 units/ml of the cytoplasm) (Tab 1) (see [11]). Thus, the absence of developmental arrest in embryos injected with SAMDC mRNA at stages later than 4-cell stage (Fig 1) could not be explained by the failure of the overexpression of SAMDC.

Developmental fate of descendant cells of the blastomere injected with SAMDC mRNA at 8-, 16-or 32-cell

Tab 1. SAMDC activities in embryos injected with SAMDC mRNA into only one of the blastomeres at different stages of development

\begin{tabular}{|c|c|c|c|c|}
\hline $\begin{array}{l}\text { mRNA } \\
\text { injection* }\end{array}$ & $\begin{array}{l}\text { Stage of injection } \\
\text { (hours post- } \\
\text { fertilization) }\end{array}$ & $\begin{array}{l}\text { Estimated volumes of } \\
\text { the cytoplasm } \\
\text { injected with mRNA } \\
(\mu \mathrm{l})\end{array}$ & $\begin{array}{c}\text { SAMDC Activity** } \\
\text { (pmoles }{ }^{14} \mathrm{CO}_{2} / \mathrm{h} / \\
\text { embryo) }\end{array}$ & $\begin{array}{l}\text { SAMDC activity calculated } \\
\text { for } 1 \text { embryo-equivalent } \\
\text { volume of the mRNA-injected } \\
\text { cytoplasm }(1 \mu \mathrm{l})\end{array}$ \\
\hline None & & 1 & 0.82 & 0.82 \\
\hline $1 \mathrm{ng}$ & 2-Cell (1.75) & $1 / 2$ & 160 & 320 \\
\hline $0.25 \mathrm{ng}$ & 8-Cell (2.75) & $1 / 8^{\#}$ & 27 & $216^{\# \#}$ \\
\hline $0.063 \mathrm{ng}$ & 32-Cell (3.75) & $1 / 32^{\#}$ & 4.5 & $144^{\# \#}$ \\
\hline
\end{tabular}

*Embryos were injected with Xenopus SAMDC mRNA at ca. $2 \mathrm{ng} / \mathrm{ml}$ cytoplasm into one animal side blastomere as explained in the text (the volume of an egg being estimated to be ca. $1 \mu \mathrm{l}$ ).

*'SAMDC activity measured at the stage 9 using 50 embryos per sample before cell dissociation became apparent.

\#These values are the possible maximal estimates, since animal side blastomeres are usually smaller than vegetal side blastomeres.

\#\#These values are the possible minimal estimates as explained in ${ }^{\#}$. 

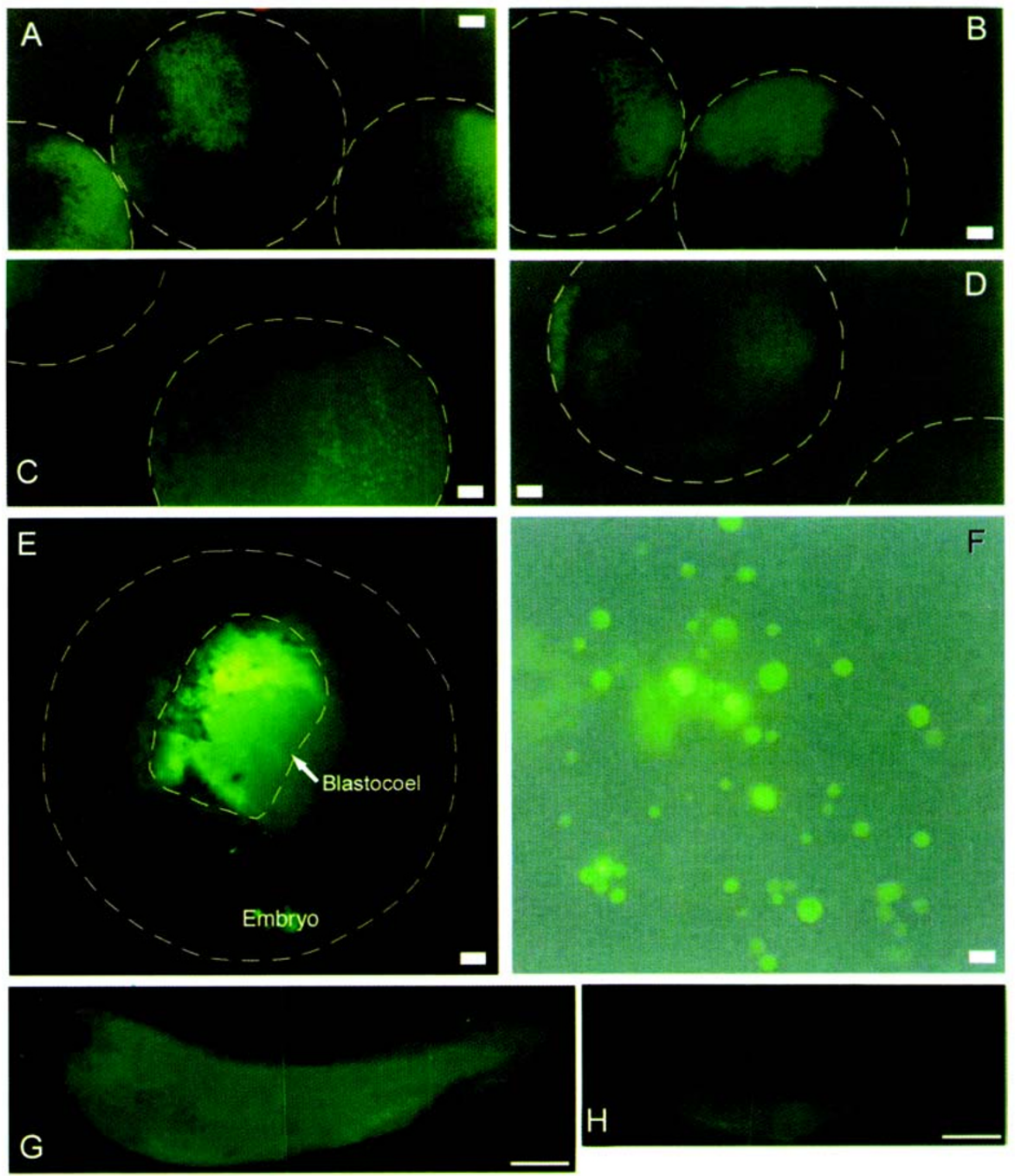

Fig 2. GFP tracing of SAMDCmRNA-injected cells at the midblastula, early gastrula and tadpole stages. Embryos were injected with $0.25 \mathrm{ng}$ of processing-defective or wild-type SAMDC mRNA togetherwith $0.025 \mathrm{ng}$ of GFP mRNA into one animal side blastomere at the 8-cell stage, and GFP luminescencewas examined at the midblastula (8h post-fertilization)(Aand B), early gastrula (12 h post-fertilization)(C-F)and tadpole (32 hpost-fertilization)(Gand H)stages. (AandB)Luminescentcellswereobserved fromtheoutsideequallyinembryos injected with the processingdefective(A)and wild-type (B)SAMDCmRNAs. Note that luminescentcells are not distributed in the wholearea, since onlyone blastomere was injected at the 8-cell stage. (C and D) At the early gastrula stage, embryos injected with processing-defective SAMDC mRNA (C) showed luminescent cells spread widely on the surface of the embryo, whereas embryos injected with wild-type SAMDC mRNA(D) showed little such luminescentcells. (E)When the embryo in (D) was stripped off the vitelline membrane and its animal cap portion was removed surgically, round luminescent cells were found abundantly in the blastocoel. The embryo looks here larger than those in (D), due to flattening caused by the absence of the vitelline membrane. $(\mathbf{F})$ When the embryo in $(E)$ was further dissected manually, luminescent dissociated cells which had been keptin the blastocoel were scattered into the medium. Scale bars indicate $0.1 \mathrm{~mm}$. (Gand $\mathbf{H})$ At the early tadpole stage, the embryo injected with mRNAforprocessing-defectiveSAMDC(G)showsstrongluminescencethroughouttheembryoaxis, theembryoinjected with wild-typeSAMDC mRNA $(\mathbf{H})$ shows little luminescence. Note that the embryo in $(\mathbf{H})$ is shorter in body length than that in $(\mathbf{G})$ due to the apoptotic loss of a certain numberofcells. Scalebars $=5 \mathrm{~mm}$ 
stage

We injected SAMDC mRNA (ca.2ng/ml cytoplasm) and GFP mRNA (ca. $0.2 \mathrm{ng} / \mathrm{ml}$ cytoplasm) together into one of the animal top blastomeres at 8-, 16or 32-cell stage, and followed the fate of cells derived from the injected blastomere by tracing the GFP signal. In this experiment, control embryos were injected with the mRNA for processing-defective Xenopus SAMDC (S70A), which like mRNA for the human processing-defective mutant SAMDC (S68A) neither increased the SAMDC activity within the embryo nor induced cell dissociation (Tab 2).

At the midblastula stage (Fig 2A, B) we found no apparent difference in the distribution of luminescent cells on the surface of embryos between the control and wild-type SAMDC mRNA-injected groups. At the early gastrula stage, widely-spread luminescent ceils occurred on the surface of the control embryos (Fig $2 \mathrm{C}$ ), but not on the surface of the embryos injected with wild-type SAMDC mRNA (Fig 2D). We then removed the animal cap from wild-type SAMDC mRNA-injected embryos, and found many round luminescent cells within their blastocoel (Fig 2E). The round luminescent cells were scattered into the medium when the embryos were manually further disrupted (Fig $2 \mathrm{~F}$ ).

When we examined the stage 9 embryos under a light microscope, many round dissociated cells were in fact found within the blastocoel (Fig 3A). Roughly, the number of the dissociated cells in the blastocoel was again dependent on the timing of injection; it decreased when the injections had been performed at later stages (data not shown). At stage 10 , the number of dissociated cells appeared to have been increased within the blastocoel (Fig $3 \mathrm{~B}$ ), and some of them seemed to have been already autolyzed (Fig 3B, arrows). Under an electron microscope, these cells were found to have fragmented nuclei (Fig 3E), and in some cells nuclear envelope was not seen. Furthermore, chromatin of such cells was extremely condensed (Fig $3 \mathrm{E}, \mathrm{F}$ ) as compared with that in the control (Fig 3D). At the neurulastage (stage 15), however, the number of abnormal round cells were not so large, and embryos appeared to be recovering from the effect of cell dissociation at the gastrula stage (Fig $3 \mathrm{C}$, arrow). Finally, at the swimming tadpole stage, we found many luminescent cells throughout the embryo axis in control embryos (Fig 2G). However, we found few luminescent cells in wild-type SAMDC-injected embryos (Fig 2H).

Based on these data, we concluded that cells derived from the SAMDC mRNA-injected blastomere are confined within the blastocoel shortly after MBT and disappear from the embryo due to apoptotic cell death, irrespective of the stage of the mRNA injection.

Embryos injected with SAMDC mRNA into a future dorsal or ventral blastomere at later stages of cleavage give rise to tadpoles with different types of anomaly

Presumptive fates of blastomeres at early cleav-

Tab 2. Development and the level of SAMDC activity in Xenopus embryos injected with mRNAs for wild-type or processing-defective mutants of Xenopus and human SAMDC

\begin{tabular}{lcccc}
\hline Injection & $\begin{array}{c}\text { Source of the } \\
\text { injected SAMDC } \\
\text { mRNA }\end{array}$ & $\begin{array}{c}\text { Number of embryos } \\
\text { used for cell } \\
\text { dissociation test }\end{array}$ & $\begin{array}{c}\text { Number of embryos } \\
\text { which underwent cell } \\
\text { dissociation }(\%)^{* *}\end{array}$ & $\begin{array}{c}\text { SAMDC activity } \\
\left(\mathrm{pmoles}^{14} \mathrm{CO}_{2} / \mathrm{h} /\right. \\
\mathrm{embryo}^{* * *}\end{array}$ \\
\hline None & Xenopus & 30 & 0 & $(0)$ \\
Wild type SAMDC & Xenopus & 20 & $20(100)$ & 0.73 \\
Processing-defective SAMDC (S70A) & & 25 & 1 & $(4)$ \\
\hline None & Human & 29 & 0 & $(0)$ \\
Wild type SAMDC & Human & 25 & $22(88)$ & 0.85 \\
Processing-defective SAMDC (S68A) & 23 & 0 & $(0)$ & 0.95 \\
\hline
\end{tabular}

*Uncleaved fertilized eggs were injected with $1 \mathrm{ng}$ of either wild type or processing-defective SAMDC mRNA.

"*Cell dissociation was examined at stage 11.

"*Determined at the stage 9 (late blastula stage) using 50 embryos for each series. This experiment was performed using

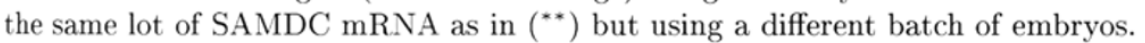



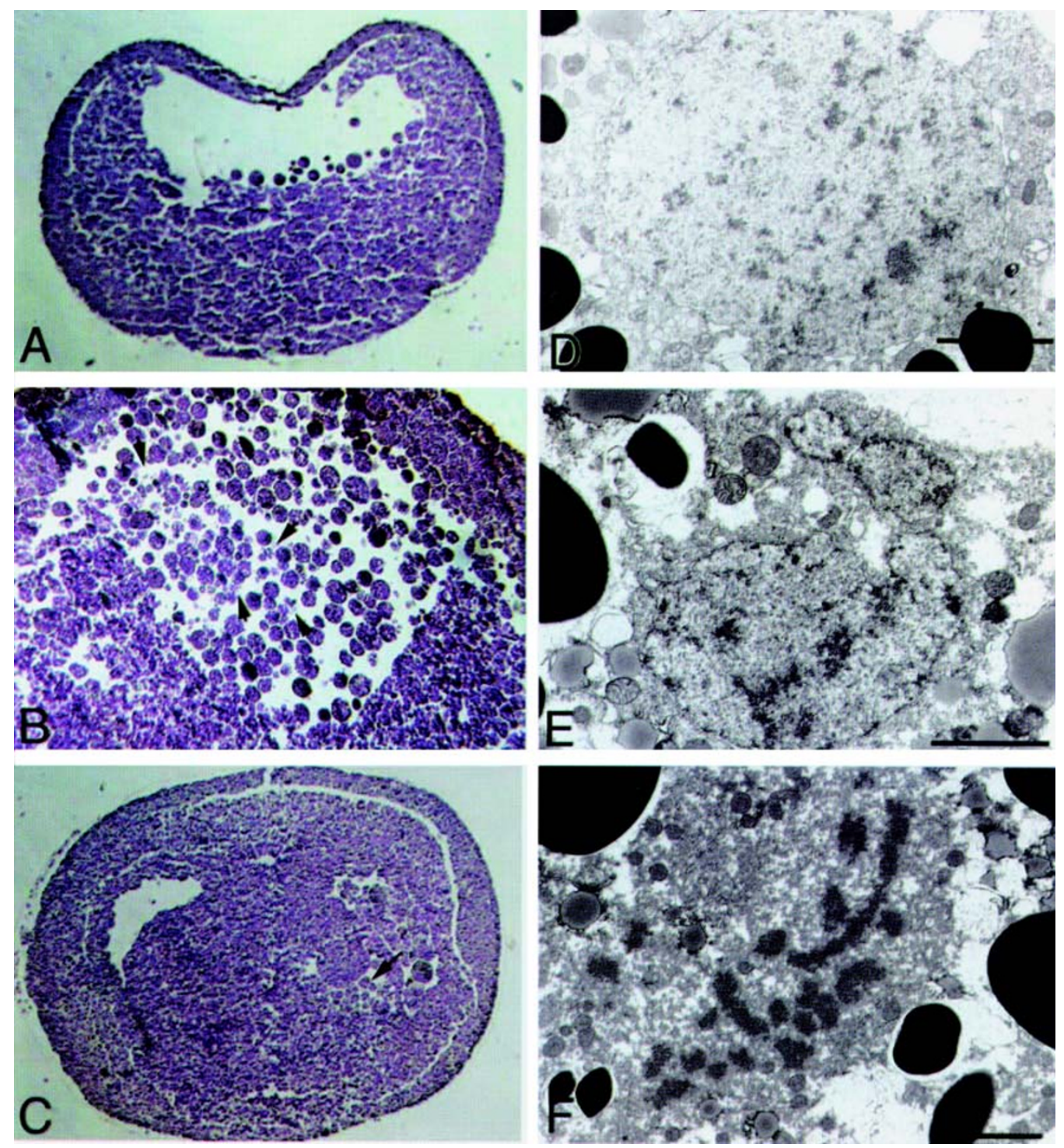

Fig 3. Lightand electron microscopic observations of SAMDC mRNA-injected embryos. (A-C)Embryos were injected with 0.013 ng of SAMDC mRNA into an animal side blastomere at 16-cell stage, fixed at the late blastula stage (stage 9)(A), early gastrula stage (stage 10)(B) orneurula stage (stage 15)(C) and examined under light microscope. (A)Round dissociated cell were observed to be confined within the blastocoel. Note that theirmembranestructures appeared to be intact.(B)Many dissociated cellswere found in the blastocoel, and some of themappeared tohave been broken (arrows) at the early gastrula stage. (C) Not many dissociated cells Were seen at the neurula stage, although there were some abnormal cells remaining (arrow).(D-F)Electronmicroscopicobservations.(D)Acontrol cell at themidgastrulastage.(E,F)Fertilized eggswere injected with $1 \mathrm{ng}$ of SAMDC mRNA, and fixed for the electron microscopy at the midgastrula stage (1 hafter the cell dissociation was observed). In the cell in (E), the nucleus is fragmented into three portions, and furthermore, chromatin is condensed. Nuclear envelope is not seen in the cell in $(F)$. Bars $=2 \mu l$

age stage could be identified by the pigmentation pattern[29, 30]. We, therefore, compared the developmental fate of embryos injected with SAMDC mRNA $(0.13 \mathrm{ng} /$ blastomere $)$ into one of the future dorsal-ani- mal or ventral-animal blastomeres at the 16-cell stage. Here again, cell dissociation accompanied with developmental arrest took place in none of the injected embryos. However, when SAMDC mRNA was injected 


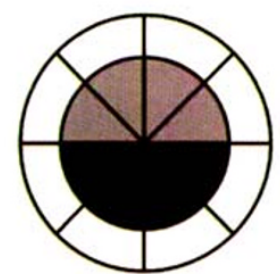

No injection

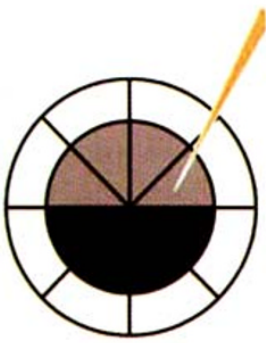

Injection to future dorsal side

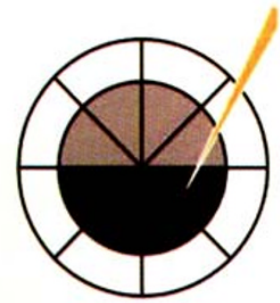

Injection to future ventral side
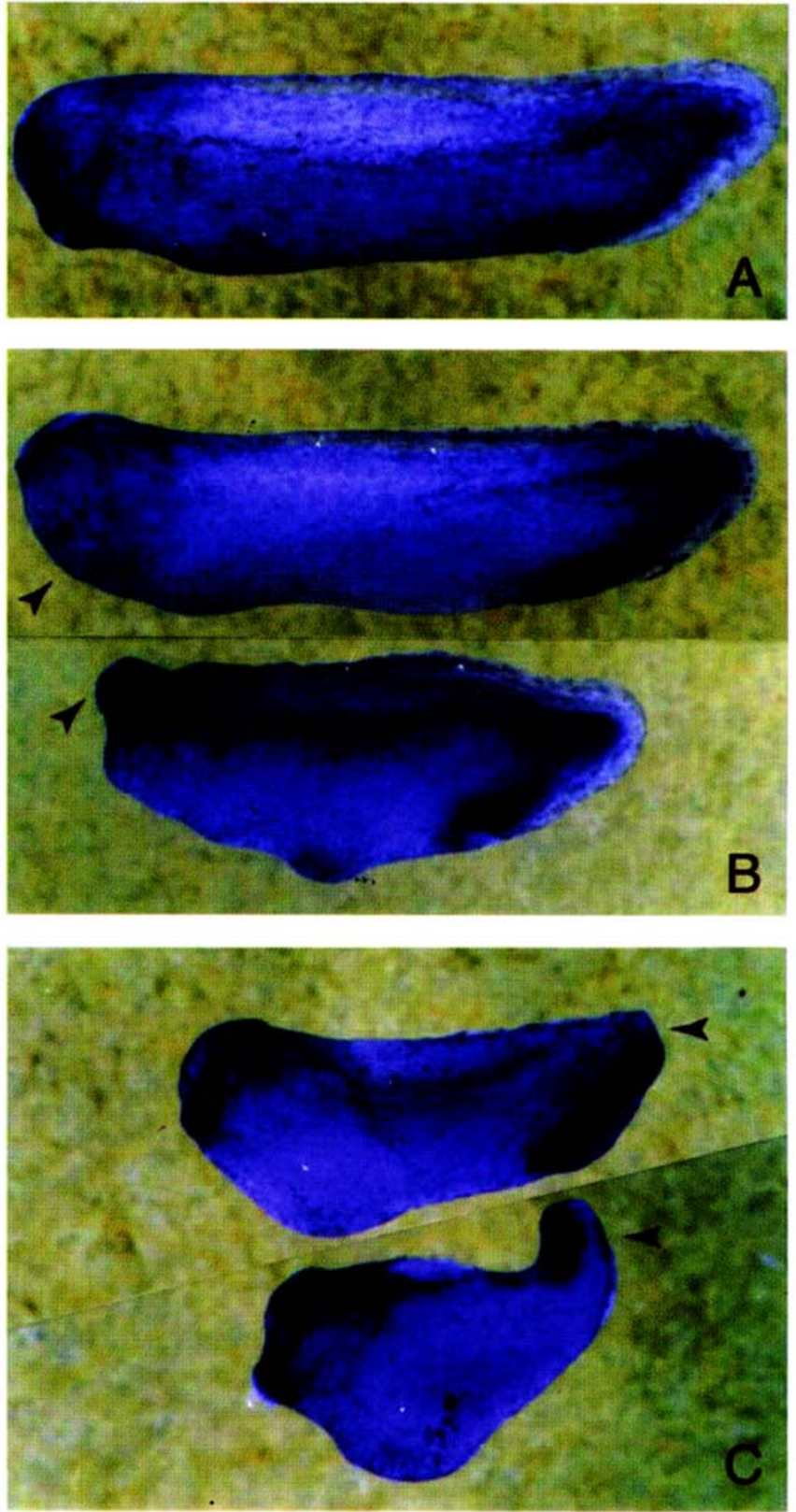

Fig 4. Effects on development of SAMDC mRNA injection into one animal side blastomere of either future dorsal or ventral side at the 16-cell stage. Embryos were injected with $0.13 \mathrm{ng}$ of SAMDC mRNA into one blastomere of either future dorsal or ventral side at 16-cell stage as schematically shown in the diagram, and cultured until the tailbud stage.(A)Acontrol uninjectedembryo.(B)Embryosinjected intothe dorsal side. The cement gland is missing (upperarrowhead), and furthermore head partis absent(acephaly)(lower arrowhead)ornotnormal. (C)Embryos injected into the ventral side. Posterior and ventral structures such as trunk and tail are poorly developed (arrowheads).

into the future dorsal animal blastomere, $38 \%$ of the injected embryos became abnormal in dorsoanterior structures (Tab 3): They formed no cement gland and/ or had defects in the head part (Fig 4B). Similarly, 27\% of the embryos injected with SAMDC mRNA into the future ventral animal blastomere developed into em- bryos abnormal in ventroposterior structures (Tab 3): They had poorly developed trunk and tail structures, although they formed normal-looking head and well developed cement gland (Fig 4C). Therefore, we concluded that the elimination of cells at the gastrula stage by SAMDC overexpression significantly affects subse- 
quent morphogenesis, reflecting the developmental fate of the eliminated cells.

\section{DISCUSSION}

Previous experiments to inject SAMDC mRNA into Xenopus embryos have been performed only at 1 - or 2-cell stage, and the outcome of the experiments was constantly deleterious and massive cell dissociation followed by whole embryo dissolution at the early gastrula stage[11, 12]. In the present experiment, we injected SAMDC mRNA into only one of the blastomeres

Tab 3. Development of the embryos injected with SAMDC mRNA into one of either future dorsal or ventral blastomere at 16-cell stage

\begin{tabular}{|c|c|c|c|c|c|}
\hline \multirow[t]{2}{*}{ Injection* } & \multirow{2}{*}{$\begin{array}{c}\text { Number of } \\
\text { embryos injected }\end{array}$} & \multirow{2}{*}{$\begin{array}{c}\text { Normal } \\
(\%)\end{array}$} & \multicolumn{3}{|c|}{ Abnormal $^{* *}$} \\
\hline & & & Defect in head part (\%) & Defect in trunk length and tail (\%) & Others (\%) \\
\hline None & 18 & $18(100)$ & $0(0)$ & $0(0)$ & $0(0)$ \\
\hline Dorsal & 13 & $7(54)$ & $5(38)$ & $0(0)$ & $1(8)$ \\
\hline Ventral & 15 & $10(67)$ & $1(7)$ & $4(27)$ & $0(0)$ \\
\hline
\end{tabular}

${ }^{*}$ Injection was performed at $0.13 \mathrm{ng} /$ blastomere at 16 -cell stage into one of either dorsal or ventral animal blastomeres.

** Anomaly of embryo was examined at stage 28.

of Xenopus embryos at different stages of cleavage ranging from 2-cell to 32-cell stages, and examined the development of the injected embryos. At first, we found that the response of embryos to the SAMDC mRNA injection differs apparently "greatly" depending on the stage of the mRNA injection: While embryos injected with SAMDC mRNA at 1- and 2-cell stages constantly underwent massive cell dissociation and stopped development at the early gastrula stage as before[11, 12], those injected with the mRNA at and after the 8-cell stage did not arrest development and often became normal-looking tadpoles. This was a puzzling result to us, since in embryos which developed into tadpoles, SAMDC was shown to be overexpressed as expected when some of the sibling embryos were sacrificed and measured for SAMDC activity at the late blastula stage. Here, the extent of the overexpression corrected for the size of the injected blastomere was just comparable to that obtained with embryos which were injected with SAMDC mRNA at 1or 2-cell stage and whose development was arrested completely at the early gastrula stage.

We then followed the fate of the cells which received SAMDC mRNA using GFP as a lineage tracer. We found here that even in embryos which were not arrested at the early gastrula stage, descendant cells of the SAMDC mRNA-injected blastomere undergo apoptotic cell death "punctually" at the early gastrula stage after being confined in the blastocoel. Based on these results, we concluded that in SAMDC mRNAinjected cells, the apoptotic program is executed "consistently" at the early gastrula stage, irrespective of the stage of the mRNA injection.

An interesting point of the present results is that such dissociated cells were first confined within the blastocoel. This is probably due to the loss of cell adhesion in such apoptosis-induced cells. It is then tempting to consider that the blastocoel provides a space for apoptotic cells to be collected and disrupted when the maternal program of apoptosis is first executed at the late blastula to early gastrula stage. Another interesting point is that when a relatively small number of cells were eliminated by the execution of the apoptotic program, embryos remain apparently intact and continue development, although such embryos often become abnormal in morphology. It appears here that the maternally-inherited program of apoptosis in Xenopus embryos eliminates damaged cells from the embryo by "altruistic cell death". In this sense the maternal program of apoptosis appears to serve as a "fail-safe" mechanism of Xenopus embryogenesis, since it eliminates damaged cells without stopping the development as a whole.

As Hensey and Gautier[14] pointed out, Xenopus embryonic cells seem to check themselves shortly after MBT to see if they are physiologically valid, when $G_{1}$ phase first appears in the cell cycle: If they find themselves physiologically aberrant, they execute the apoptotic program and disappear at cellular level but not as the embryo as a whole. The points to be checked here seem to be diverse, including intactness of DNA structure, occurrence of normal DNA replication, normal RNA transcription, and normal translation, as can be expected from the results ob- 
tained with g-Iray[13, 14], hydroxyurea[15], a-lamanitin $[14,15]$, and cycloheximide[14, 16]. Also, Xenopus embryonic cells seem to check if DNA methylation is normal or not, and if it is not normal they execute the same apoptotic program[18, 19]. These situations could be summarized as in Fig 5 , in which the apoptotic program is expressed as a "fail-safe" mechanism of normal Xenopus embryonic development.

\section{ACKNOWLEDGMENTS}

We thank Professor A. E. Pegg (Pennsylvania
State University) for providing us with plasmids for human SAMDC and its processing-defective mutant S68A. We also thank Professor J. B. Gurdon (Cambridge University) for providing us with the plasmid for GFP. Special thanks are due to Dr. Yoshio Yaoita for valuable discussions especially on the experiment Fig 1.

\section{REFERENCES}

1 Shiokawa K, Kurashima R, Shinga J. Temporal control of gene expressionfromendogenousandexogenously-introducedDNAs

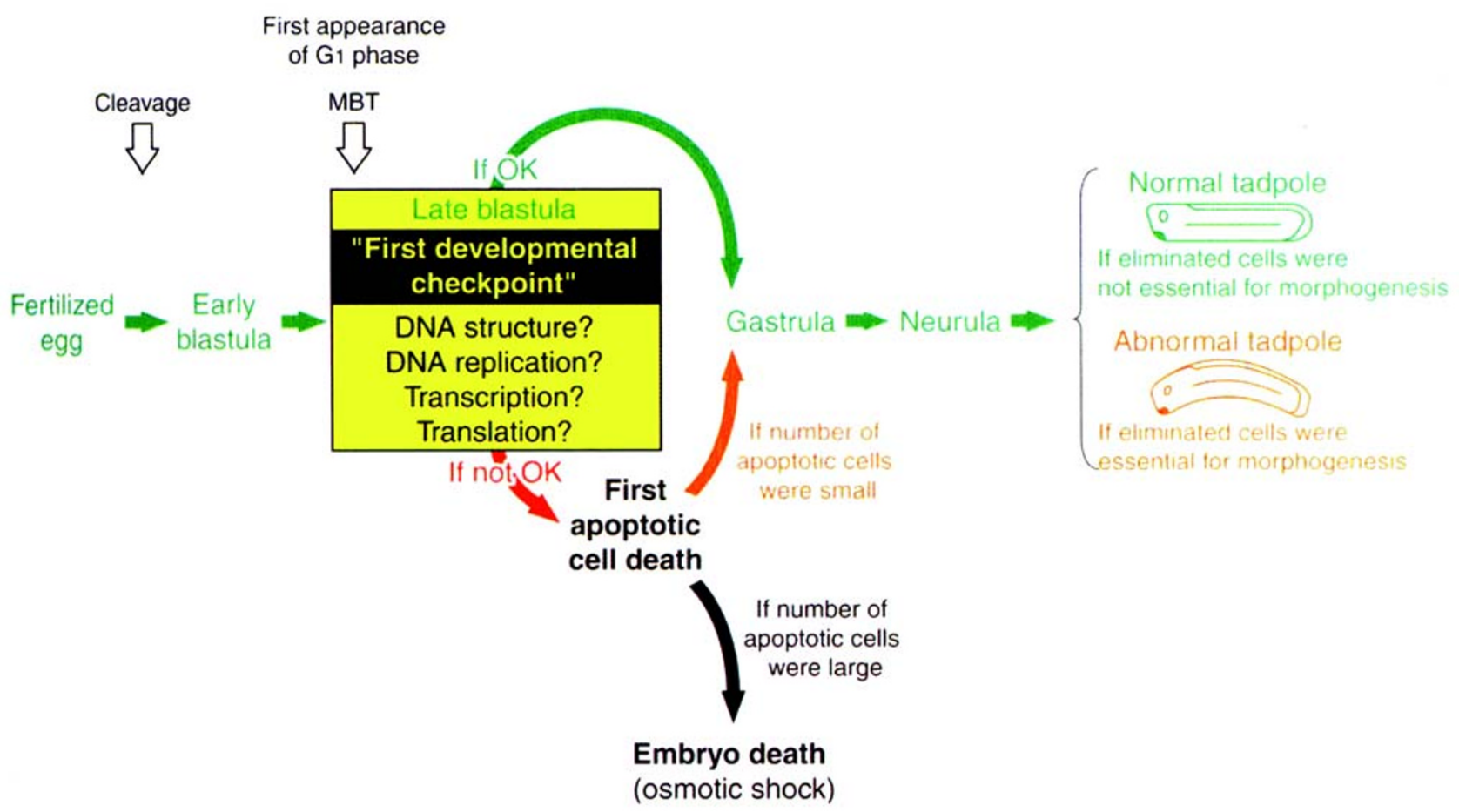

Fig 5. A model which shows how early development proceeds. This model includes apoptotic check point which seems to function as a part of the surveillance and "fail-safe"mechanism in Xenopus earlyembryonic development. The fertilized egg cleaves rapidly until itreaches theearly blastula stage. Shortly after MBT, the "first developmental checkpoint" comes when $\mathrm{G}_{1}$ phase first appears and this check mechanism cellautonomously determines if the cell should continue on developmentor be eliminated by executing the maternal apoptosis program. However, even when the apoptotic program was set on operation, embryos could follow two different courses. If the number of apoptotic cells was large, the whole embryo stops development and dies, since apoptotic cells are dissociated and autolyzed due to osmotic shock (eggs are laid in the water). However, ifthenumber of apoptotic cells is small, they are confined within the blastocoeland theembryo itselfcontinues on development. Tadpoles which come out from such survived embryos may be normal. However, when the eliminated cells are too many or indispensable for normalmorphogenesis, embryos developintoabnormaltadpoles.

in early embryogenesis of Xenopus laevis. Int J Dev Biol 1994; 38:249-55.

2 Woodland HR. Changes in the polysome content of developing Xenopus laevis embryos. Dev Biol 1974; 40: 90-101.
3 Davidson EH. Gene Activity in Early Development. Academic Press: New York 1976.

4 Graham CF, Morgan RW. Changes in the cell cycle during early amphibian development. DevBiol 1966;14:439-60.

5 NewportJ,KirschnerM.Amajordevelopmental transitioninearly Xenopus embryos: I. Characterization and timing of cellular 
changes at the midblastula stage. Cell 1982; 30:675-86.

6 Almouzni G, Khochbin S, DimitrovS, Wolffe AP. Histone acetylation influences both gene expression and development of Xenopus laevis. Dev Biol 1994; 165:654-69.

7 SignoretJ, Lefresne J. Contribution àl'étude de la segmentation de l'oeuf d'axolotl. II. Influence de modifications du noyau et du cytoplasme sur les modalit's de la segmentation. Ann Embryol Morphol 1973;6:299-307.

8 Minoura I, Nakamura H, Tashiro K, Shiokawa K. Stimulation of circus movement by activin, bFGF and TGFbeta 2 in isolated animal cap cells of Xenopus laevis. Mech Dev 1995; 49:65-9.

9 Shiokawa K, Misumi Y, Yamana K. Demonstration of rRNA synthesis in pre-gastrulation embryos of Xenopuslaevis. Dev Growth Differ 1981; 23:579-87.

10 Nakahashi T, Yamaha K. Biochemical and cytological examination of the initiation of ribosomal RNA synthesis during gastrulation of Xenopus laevis. Dev Growth Differ 1976; 18:329-39.

11 Shibata M, Shinga J, Yasuhiko Y, et al. Overexpression of S-adenosylmethionine decarboxylase (SAMDC) in early Xenopus embryos induces cell dissociation and inhibits transition from the blastula to gastrula stage. Int J Dev Biol 1998; 42:675-86.

12 Kai M, Higo T, Yokoska J, et al. Overexpression of Sadenosylmethionine decarboxylase (SAMDC) activates the maternal program of apoptosis shortly after MBT in Xenopus embryos. Int J Dev Biol 2000; 44:507-10.

13 Anderson JA, Leweltyn AL, Mailer JL. Ionizing radiation induces apoptosis and elevates cyclin A1-Cdk2 activity before but not after the midblastula transition in Xenopus. Mol Biol Cell 1997; 8:1195-206.

14 Hensey C. Gautier J. A develoPmental timer that regulates apoptosis at the onset of gastrulation. Mech Dev 1997; 69:183-95.

15 Stack JH, Newport JW. Developmentally regulated activation of apoptosis early in Xenopus gastrulation results in cyclin A degradation during interphase of the cell cycle. Development 1997; 124:3185-95.

16 Sible JC, Anderson JA, Lewelly AL, Mailer JL. Zygotic Transcription is required to blockamaternal program ofapoptosis in Xenopus embryos. DevBiol 1997; 189:335-46.

17 Hensey C, Gautier J. Programmed cell death during Xenopus Development: Aspatial-temporal analysis. Dev Biol 1998; 203: 36-48.

18 Kaito C, Kai M, Higo T, et al. Activation of the maternally preset program of apoptosis by microinjection of 5-aza-2'deoxycytidineand5-methyl-2'-deoxycytidine-5'-triphosphasein Xenopus laevis embryos. Dev Growth Differ 2001;43:383-90.

19 Stancheval, Hensey C, Meehan RR. Loss of the maintenance methyltransferase, $\mathrm{xDnmtl}$, induces apoptosis in Xenopus embryos. EMBOJ2001;20:1963-73.

20 Carter AD, Sible JC. Loss of XChkl function triggers apoptosis afterthemidblastulatransition in Xenopuslaevisembryos. Mech Dev2003; 120:315-23.
21 Ikegami R, HunterP, Yager TD. Developmental activation of the capability to undergo checkpoint-induced apoptosis in the early zebrafishembryo. DevBiol 1999; 209:409-33.

22 Finkielstein CC, Lewellyn AL, Maller JL. The midblastula transition in Xenopus embryos activates multiple pathways to prevent apoptosis in response toDNAdamage. ProcNatl Acad Sci2001; 98:1006-11.

23 Shinga J, Kashiwagi K, Toshiro K, Igarashi K, Shiokawa K. Maternal and zygotic expression of mRNA for Sadenosylmethionine decarboxylase and its relevance to the unique polyamine composition in Xenopus oocytes and embryos. Biochim Biophys Acta 1996; 1308:31-40.

24 Stanley BA, Shantz LM, Pegg AE. Expression of mammalian S-adenosylmethionine decarboxylase in Escherichia coll. Determination of sites for putrescine activation of activity and processing. J Biol Chem 1994; 269:7901-7.

25 Stanley BA, Pegg AE. Amino acid residues necessary for putrescine stimulation of human $\mathrm{S}$-adenosylmethionine decarboxylase proenzyme processing and catalytic activity. J Biol Chem 1991; 266:18502-6.

26 Zernicka-Goetz M, Pines J, Ryan K, et al. An indelible lineage marker for Xenopus using a mutated green fluorescent protein. Development 1996; 122:3719-24.

27 Koga C, Adati N, Nakata K, et al, Characterization of a novel member of the FGF family, XFGF-20, in Xenopus laevis. Biochem Biophys Res Comm 1999; 261:756-65.

28 Nieuwkoop PD, Faber J. Normal Table of Xenopus laevis (Daudin). Elsevier: Amsterdam 1967.

29 Shiokawa K, Yamana K. Differential initiation of rRNA gene activity in progenies of different blastomeres of early Xenopus embryos: evidence for regulated synthesis of rRNA. Dev Growth Differ 1979; 21:501-7.

30 Moody SA. Fates of the Blastomeres of the 16-Cell Stage XenopusEmbryo. DevBiol 1987; 119:560-78. 